\title{
Case Report \\ Oral Capecitabine Achieves Response in Metastatic Eccrine Carcinoma
}

\author{
Kristian Larson $\mathbb{D}^{\mathbb{D}},{ }^{1}$ Hani M. Babiker ${ }^{(D)},{ }^{1,2,3}$ Andrew Kovoor, ${ }^{3}$ Joy Liau, ${ }^{4}$ Jordan Eldersveld, ${ }^{5}$ \\ and Emad Elquza ${ }^{1,3}$ \\ ${ }^{1}$ Early Phase Clinical Trial Program, The University of Arizona Cancer Center, 1515 N. Campbell Ave., Tucson, AZ 85724, USA \\ ${ }^{2}$ Translational Genomics Research Institute (TGen), 445 N. 5th Street, Phoenix, AZ 85004, USA \\ ${ }^{3}$ Division of Hematology and Oncology, University of Arizona College of Medicine, 1515 N. Campbell Ave., Tucson, \\ AZ 85724, USA \\ ${ }^{4}$ Department of Medical Imaging, University of Arizona, 1501 N. Campbell Ave., Tucson, AZ 85712, USA \\ ${ }^{5}$ Department of Pathology, University of Arizona, 1501 N. Campbell Ave., Tucson, AZ 85712, USA
}

Correspondence should be addressed to Kristian Larson; kris7c@email.arizona.edu

Received 14 September 2017; Accepted 21 January 2018; Published 1 March 2018

Academic Editor: Jose I. Mayordomo

Copyright ( $\odot 2018$ Kristian Larson et al. This is an open access article distributed under the Creative Commons Attribution License, which permits unrestricted use, distribution, and reproduction in any medium, provided the original work is properly cited.

\begin{abstract}
The low prevalence rate and limited literature on eccrine carcinoma (EC) pose a challenge to properly diagnosing and treating this rare malignancy. EC lesions tend to present similarly to other cutaneous neoplasms and dermatitis-like conditions. Efficacious treatment guidelines have not been established for patients diagnosed with EC, and few treatment regimens have demonstrated clinical benefit. Due to the high metastatic potential of EC, recognizing the clinical presentation, properly diagnosing, and utilizing beneficial treatment options are important for managing this disease. We report a case of a 66-year-old female who presented with lesions that her primary care provider misdiagnosed as basal cell carcinoma. The disease responded poorly to taxane- and platinum-based chemotherapies as well as an isolated limb perfusion of an alkylating agent. However, continuous dosing of oral capecitabine achieved an 18-month period of progression free survival (PFS) and ameliorated quality of life. We wish to highlight this rare disease and discuss presentation, diagnosis, and management as it is most often misdiagnosed leading to advanced metastatic disease when patients present to the oncologist. In addition, it is crucial to study and report potentially efficacious regimens considering the lack of clinical trials in this disease.
\end{abstract}

\section{Introduction}

Eccrine carcinoma (EC) is an appendageal cancer that originates from the eccrine sweat gland and accounts for an estimated $0.005-0.01 \%$ of diagnosed cutaneous malignancies [1]. Histopathologies closely associated with EC include hidroadenocarcinoma, spiradenocarcinoma, and porocarcinoma [1-3]. A consistent clinical presentation of these lesions has not been identified; other authors have reported brown, bluish, and erythematous lesions which may appear papular or ulcerative $[1,4,5]$. Proper recognition and diagnosis of EC prove to be difficult due to its infrequency and similar presentation to basal and squamous cell carcinoma, amelanotic melanoma, seborrheic keratosis, cutaneous lymphoma, and verruca vulgaris [1].
EC lesions are commonly reported in the lower extremities (35\%), head and neck (24\%), and upper extremities (14\%) [1]. Immunohistochemical (IHC) stains and serum cancer markers have also been inconsistently reported, which include but are not limited to carcinoembryonic antigen, epithelial membrane antigen, estrogen receptors, progesterone receptors, cytokeratin 7 , and pancytokeratins $[2,6]$. Thus, recognizing the pleomorphic features of basaloid cells within the epidermis that infiltrate into the ductal papillary structures of the dermis help diagnose EC [2].

A limited understanding of EC in addition to unestablished treatment guidelines contributes to a relative mortality rate of $80 \%$ and a 10 -year disease survival rate of $9 \%$ when metastatic in stage $[1,7]$. Based on the current literature, there seems to be 


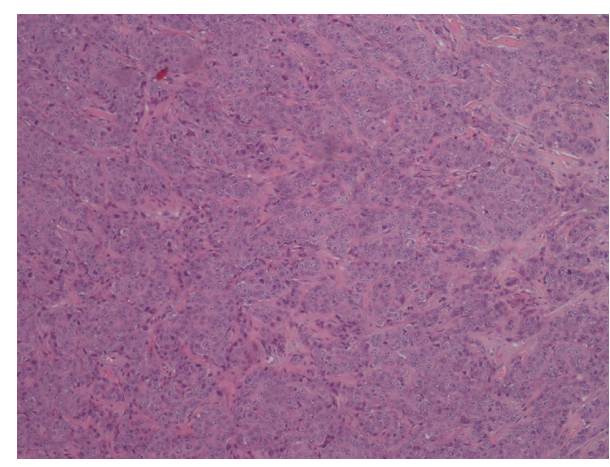

FIgURE 1: Eccrine carcinoma. Infiltrative, poorly differentiated neoplasm in a nested to trabecular pattern. Nuclei are relatively uniform with notably prominent nucleoli (hematoxylin-eosin, original magnification $\times 200$ ).

a clinical consensus that wide surgical excision with the goal of clear margins is the most effective treatment for locoregional EC [8]. However, this option is not optimal for metastatic cases [9]. Other cases of metastatic EC have illustrated resistance to radiotherapy as well as a variety of chemotherapies [9]. We describe a case of a patient who continued to progress during treatments with carboplatin, paclitaxel, melphalan, actinomycin $\mathrm{D}$, and radiotherapy but achieved 18 months of progression free survival (PFS) and an improved quality of life with oral capecitabine despite her diffusely metastatic disease.

\section{Case Description}

A 66-year-old female with a history of ongoing thrombocytosis (platelets at $724 \times 10^{3} / \mathrm{uL}$, range: $150-450 \times 10^{3} / \mathrm{uL}$ ), emphysema and coronary artery disease visited her primary care physician (PCP) with complaints about pruritic "bumps" on her left calf in 2007. Her PCP noted that the lesions presented as erythematous, $2-3 \mathrm{~mm}$ in diameter, and papular. The PCP diagnosed these neoplasms as basal cell carcinoma without further diagnostic testing or intervention. The patient returned in early 2009 with enlarged lesions, increased pruritus, and ulceration. The patient was referred to dermatology and oncology.

She underwent wide surgical excision with clear margins attained, and three lymph nodes from the left inguinal zone were excised in April 2009. One lymph node was positive for disease involvement. A skin graft was placed thereafter. Histopathological analysis of the skin resection (Figure 1) supported the initial diagnosis of EC. Immunohistochemistry (IHC) resulted negative for the expression of estrogen and progesterone receptors and positive for HER-2/neu and EGF-R (2+ on 0-3+ scale). Albeit the aforementioned IHC data have been reported in EC, there are inconsistent and inadequate data in literature confirming it as a diagnostic tool. Unremarkable CT studies warranted no remaining disease.

The patient returned in July 2009 with recurrent erythematous papular lesions throughout the skin graft of the left calf and new multifocal lesions in transit with the left medial thigh. A punch biopsy of the left thigh demonstrated recurrent metastatic EC. The metastatic lesion resulted negative for the aforementioned positive IHC stains on the primary

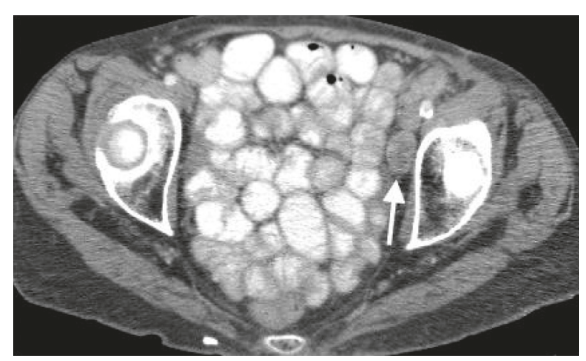

(a)

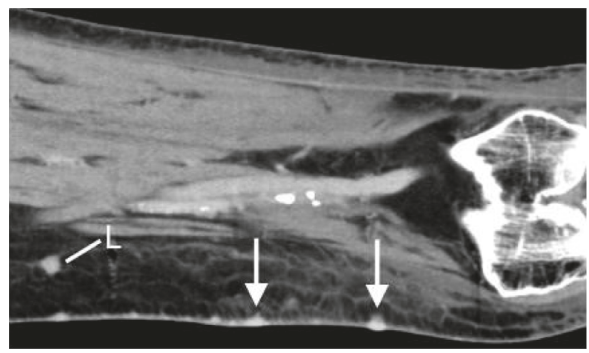

(b)

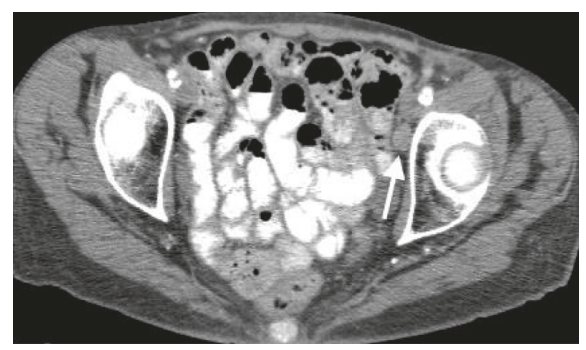

(c)

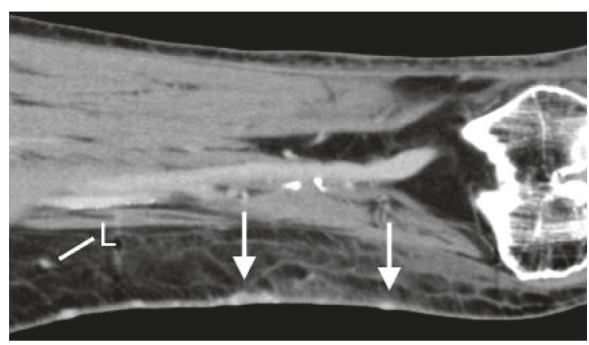

(d)

Figure 2: Progression of the disease despite treatment with carboplatin, paclitaxel, and radiotherapy ( $a, b)$, with subsequent partial treatment response two months after starting capecitabine (c, d). Axial postcontrast CTs of the pelvis show metastatic left pelvic lymph nodes, with decrease in size of a metastatic left external iliac chain lymph node from $2.2 \times 1.1 \mathrm{~cm}$ (white arrow, a) to $1.5 \times 0.9 \mathrm{~cm}$ (white arrow (c)) with capecitabine treatment. Coronal postcontrast CTs of the left leg (b) before and (d) after capecitabine treatment show similar cutaneous metastases (white arrows) with decreased size of a metastatic lymph node (L).

tumor. CT studies indicated no internal organ metastasis. Surgical oncology performed a hypoxic-hyperthermic isolated limb perfusion on the left leg with melphalan and actinomycin D that resulted in a partial response. However, the patient returned in December 2009 with recurrent lesions along her left thigh and calf measuring 1-2 centimeters in diameter and appearing ulcerated. In March 2010, a regimen 


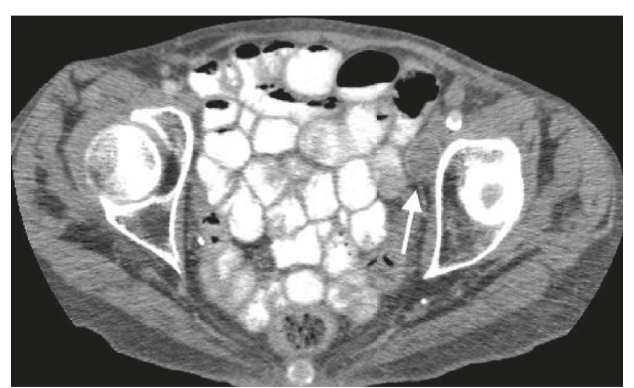

(a)

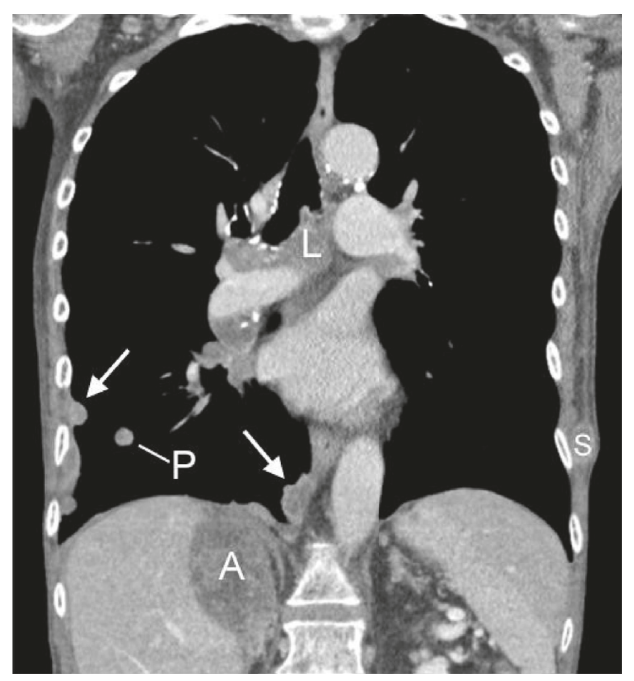

(c)

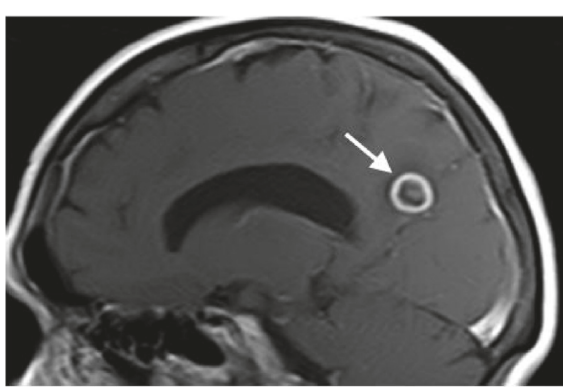

(b)

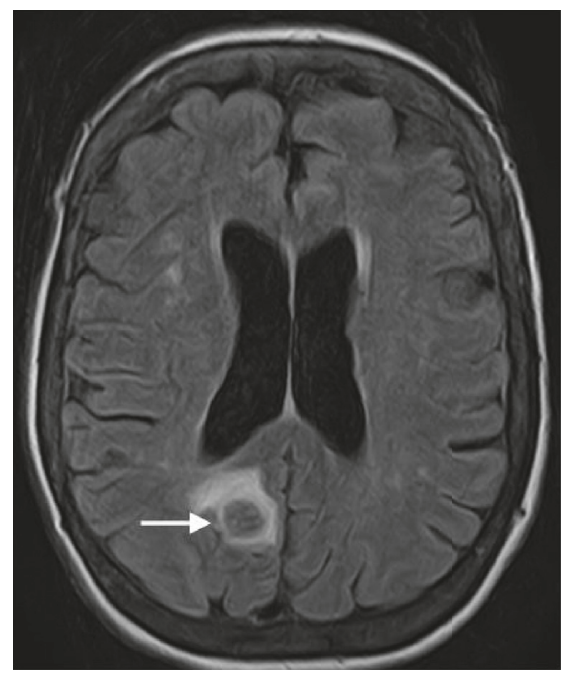

(d)

Figure 3: Progression of disease 18 months after starting capecitabine treatment. Axial postcontrast CT of the pelvis (a) shows increased size of a centrally necrotic left external chain lymph node, measuring $2.2 \times 1.7 \mathrm{~cm}$ (white arrow). Postcontrast sagittal T1 MRI (b) and noncontrast coronal FLAIR MRI (d) of the brain show a new rim enhancing $1.2 \mathrm{~cm}$ metastasis in the right posterior parietal lobe with surrounding edema (white arrows). Coronal postcontrast CT of the chest and abdomen (c) shows new right pleural metastasis (arrows), mediastinal and hilar lymph node (L), and left lateral chest wall soft tissue (S) metastases, as well as an enlarging right lower lobe pulmonary metastasis $(\mathrm{P})$ and right adrenal metastasis superimposed on an existing adrenal adenoma (A).

of intravenous carboplatin (AUC of 2 ) and paclitaxel $\left(45 \mathrm{mg} / \mathrm{m}^{2}\right.$ ) given on days 1,8 , and 15 (28-day cycle) concurrent to sensitizing $6 \mathrm{MV}$ external beam radiation (4500 cGy over 25 fractions applied to the left extremity lesions) was performed, and she completed two cycles of therapy by May 2010. CT scans in October 2011 revealed left pelvic lymph node metastases (Figure 2(a)) as well as recurrence in the left leg (Figure 2(b)).

In February 2012, the patient started a capecitabine regimen (1500 mg PO q12h) that followed a 2-week on/1-week off dosing schedule. Due to grade 3 palmar-plantar erythrodysesthesia syndrome occurring 12 months after dosing, the dose was reduced to $1000 \mathrm{mg}$. The absence of cutaneous lesion recurrence was noted. CT studies (Figure 2) revealed an overall partial response with a decrease in size of the left pelvic lymph node metastases (Figure 2(c)) and cutaneous lesions in the left leg (Figure 2(d)). Capecitabine was continued until August 2013 when new cutaneous metastases appeared in the left gluteus $(1.3 \mathrm{~cm})$ and right anterior breast $(1.7 \mathrm{~cm}) . \mathrm{CT}$ studies showed interval enlargement of left pelvic lymph node, right adrenal, and pulmonary metastases (Figures 3(a) and 3(c)). New metastatic disease was identified in the thoracic and abdominal lymph nodes, the right pleura, and cutaneous tissue (Figure 3(c)). An MRI of the brain revealed multiple metastases with index lesions in the right posterior parietal lobe (Figures 3(b) and 3(d)). The patient was referred to palliative care and hospice; she expired shortly after.

\section{Discussion}

Treatment strategies have been reported (Table 1) and illustrate the resistant nature of EC to numerous chemotherapies with only a few options increasing patient survival. Patients treated with platinum-based, taxane-based, and tyrosine kinase inhibitors seem not to derive a substantial therapeutic benefit. The tyrosine kinase inhibitor, sunitinib, achieved 8 months of PFS after a patient progressed through prior platinum-based therapy [10]. Poor outcomes are also noted with the anthracycline antibiotic, doxorubicin, and monotherapy with cyclophosphamide; EC rapidly progressed with metastases into multiple organs and the patients expired soon after $[4,11]$.

Distinct tumor markers have not been characterized for $\mathrm{EC}$, and other authors have reported a variety of positive IHC stains $[2,6,13]$. Thus, correlative studies of biomarkers can help develop targeted therapies aimed at specific pathways and 
TABLE 1: Demographics, presentations, treatments, and outcomes of reported cases of metastatic EC.

\begin{tabular}{|c|c|c|c|c|c|}
\hline $\begin{array}{l}\text { Serial } \\
\text { number }\end{array}$ & $\begin{array}{c}\text { Age/sex } \\
\text { [reference] }\end{array}$ & $\begin{array}{l}\text { Presentation of primary } \\
\text { cutaneous lesion }\end{array}$ & Metastatic sites & Treatment & Outcome \\
\hline 1 & $60 / \mathrm{F}[5]$ & Ulcerative, nodular, scaly, erythematous & Axillary and subclavicular LNs & Capecitabine & PFS (18 months) \\
\hline 2 & $73 / \mathrm{M}[12]$ & Ulcerative, nodular, erythematous & Parotid gland, cervical LNs, lung & Capecitabine & CR \\
\hline 3 & $64 / \mathrm{F}[13]$ & ND & Intraparotid LN & Tamoxifen & PFS (3 years) \\
\hline 4 & $43 / \mathrm{F}[10]$ & ND & $\begin{array}{c}\text { Occipital LNs, cervical nerve root, } \\
\text { vertebrae }\end{array}$ & Sunitinib & PFS (8 months) \\
\hline 5 & $45 / \mathrm{F}[11]$ & ND & Cervical LNs, bone, choroid & Doxorubicin & $\begin{array}{l}\text { POD (deceased } \\
\text { after } 2 \text { months) }\end{array}$ \\
\hline 6 & $59 / \mathrm{F}[4]$ & Bluish nodule, $2 \mathrm{~cm}$ diameter & Mediastinal LNs, lung & Cyclophosphamide & $\begin{array}{l}\text { POD (deceased } \\
\text { after } 1 \text { month) }\end{array}$ \\
\hline
\end{tabular}

CR: complete response; F: female; LN: lymph node; M: male; ND: not described; PFS: progression free survival; POD: progression of disease.

genetic mutations $[2,13]$. For example, a case was reported of a patient with metastatic EC whose tumor biopsy revealed $100 \%$ reactivity for estrogen and progesterone receptors; the patient was started on empirical tamoxifen citrate therapy and achieved 3 years of complete remission [13]. On the other hand, our patient had negative reactivity for both of these receptors, so molecular profiles need to be identified and the prevalence assessed. A study that evaluated the molecular profiles of apocrine-eccrine carcinoma cases found that 6 of the 7 EC cases tested positive for the epidermal growth factor receptor (EGFR) mutant [14]. This highlights a potential role for clinical trials to determine the outcomes of targeted therapies that correspond to pertinent oncogene mutations [14].

To the best of our knowledge, we report the third case of metastatic EC treated with capecitabine $[5,12]$. Our patient continued on capecitabine dosing from February 2012 to August 2013 without disease progression. Per RECIST version 1.1 criteria, our patient's follow-up CT study (Figures 2(c) and 2(d)) showed a partial response two months after starting capecitabine treatment. This regimen of oral capecitabine afforded efficacy and improved the patient's quality of life compared to the doublet therapy of carboplatin and paclitaxel which had more toxicities. The high mortality and morbidity rates associated with metastatic EC indicate the necessity for clinical trials to fully delineate safety and efficacy of potential future therapies [1-3, 9].

\section{Conclusion}

Eccrine carcinoma is an uncommon skin cancer that accompanies a poor prognosis. The clinical presentation of EC can be equivocal and result in misdiagnosis, which adversely affects patient outcomes. Standard of care treatment options for metastatic EC have not yet been developed. Further investigation into the efficacy of capecitabine treatment as monotherapy or in combination with other regimens for metastatic EC is worthwhile to improve the poor outcomes of diagnosed patients. Moreover, correlation of molecular profiles to tumor responses would provide helpful data to identify efficacious treatment regimens in the future.

\section{Conflicts of Interest}

The authors declare that they have no conflicts of interest.

\section{Acknowledgments}

The authors thank oncologic pharmacist, Ali McBride, PharmD, for providing data on drug dosing.

\section{References}

[1] A. M. Salih, F. H. Kakamad, R. A. Essa et al., "Porocarcinoma: a systematic review of literature with a single case report," International Journal of Surgery Case Reports, vol. 30, pp. 13-16, 2017.

[2] A. Gómez-Zubiaur, S. Medina-Montalvo, M. D. Vélez-Velázquez, and I. Polo-Rodríguez, "Eccrine porocarcinoma: patient characteristics, clinical and histopathologic features, and treatment in 7 cases," Actas Dermo-Sifiliográficas, vol. 108, no. 4, pp. e27-e32, 2017.

[3] S. N. Tolkachjov, A. R. Schmitt, J. G. Muzic, A. L. Weaver, and C. L. Baum, "Incidence and clinical features of rare cutaneous malignancies in Olmsted County, Minnesota, 2000 to 2010," Dermatologic Surgery, vol. 43, no. 1, pp. 116-124, 2017.

[4] V. K. Dave, "Eccrine sweat gland carcinoma with metastases," British Journal of Dermatology, vol. 86, no. 1, pp. 95-97, 1972.

[5] T. Jouary, A. Kaiafa, P. Lipinski et al., "Metastatic hidradenocarcinoma: efficacy of capecitabine," Archives of Dermatology, vol. 142, no. 10, pp. 1366-1367, 2006.

[6] G. Sanchez Petitto, N. M. Sarwari, P. Jain, M. Swaby, and M. Bhattacharjee, "FDG PET/CT in malignant eccrine spiradenoma," Clinical Nuclear Medicine, vol. 42, no. 2, pp. 125-126, 2017.

[7] Chintamani, R. D. Sharma, R. Badran, V. Singhal, S. Saxena, and A. Bansal, "Metastatic sweat gland adenocarcinoma: a clinicopathological dilemma," World Journal of Surgical Oncology, vol. 1, p. 13, 2003.

[8] M. Shaw, P. H. McKee, D. Lowe, and M. M. Black, "Malignant eccrine poroma: a study of twenty-seven cases," British Journal of Dermatology, vol. 107, no. 6, pp. 675-680, 1982.

[9] C. J. Coonley, D. P. Kelsen, A. G. Huvos, P. Schauer, and P. Sordillo, "Chemotherapy of metastatic sweat gland carcinoma. A retrospective review," American Journal of Clinical Oncology, vol. 8, no. 4, pp. 307-311, 1985.

[10] M. Battistella, C. Mateus, N. Lassau et al., "Sunitinib efficacy in the treatment of metastatic skin adnexal carcinomas: report of two patients with hidradenocarcinoma and trichoblastic carcinoma," Journal of the European Academy of Dermatology and Venereology, vol. 24, no. 2, pp. 199-203, 2010.

[11] L. R. Moura, B. Ayres, L. Feijóo, S. Romano, A. N. Odashiro, and M. N. Burnier Jr., "Presumed eccrine carcinoma 
metastatic to the choroid," Clinical and Experimental Ophthalmology, vol. 34, no. 7, pp. 699-700, 2006.

[12] A. Lerner, A. Beckford, S. Ugent, L. Goldberg, S. Jalisi, and M. F. Demierre, "Complete response of metastatic malignant hidradenocarcinoma to capecitabine treatment," Archives of Dermatology, vol. 147, no. 8, pp. 998-999, 2011.

[13] U. Schröder, V. Dries, J. P. Klussmann, C. Wittekindt, and H. E. Eckel, "Successful adjuvant tamoxifen therapy for estrogen receptor-positive metastasizing sweat gland adenocarcinoma: need for a clinical trial?," Annals of Otology, Rhinology \& Laryngology, vol. 113, no. 3, pp. 242-244, 2004.

[14] L. P. Le, D. Dias-Santagata, A. C. Pawlak et al., "Apocrineeccrine carcinomas: molecular and immunohistochemical analyses," PLoS One, vol. 7, no. 10, article e47290, 2012. 


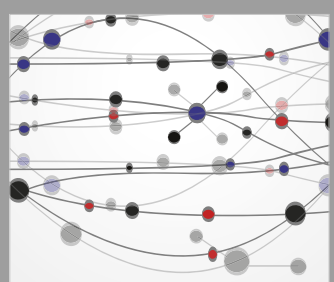

The Scientific World Journal
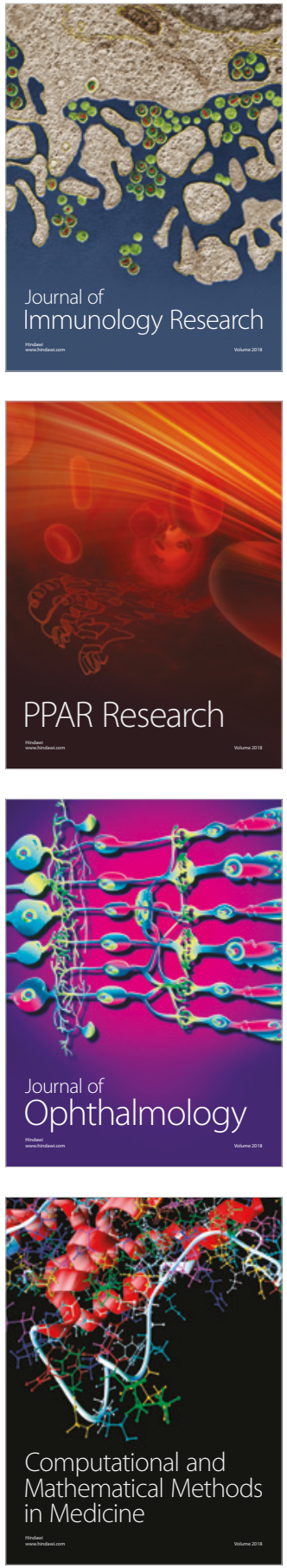

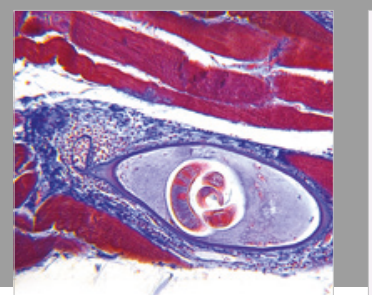

Gastroenterology Research and Practice

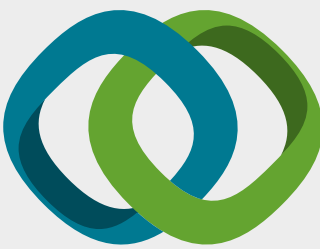

\section{Hindawi}

Submit your manuscripts at

www.hindawi.com
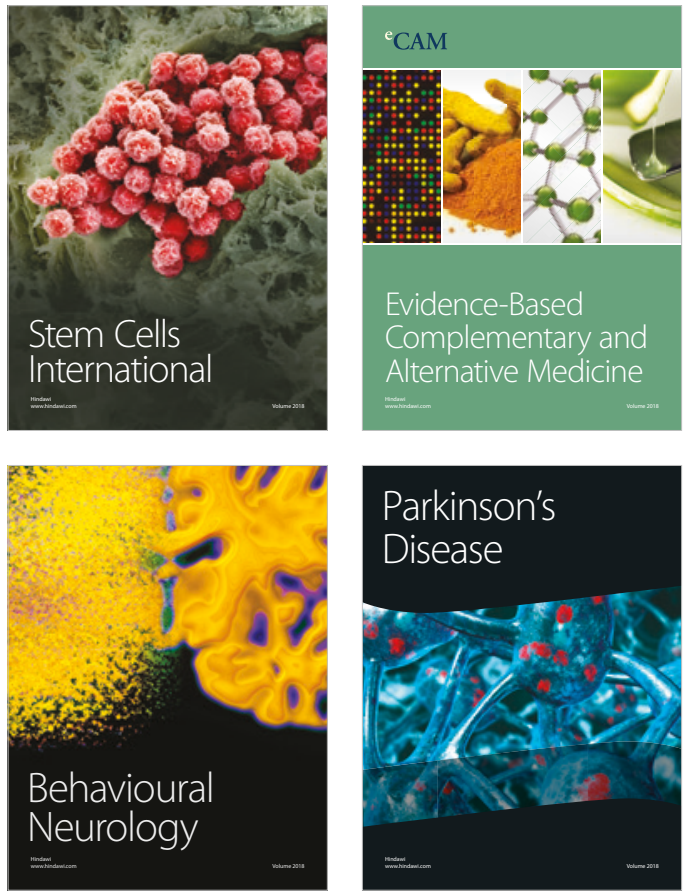

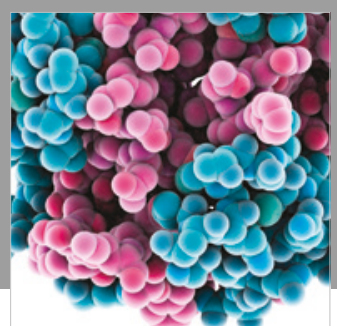

ournal of

Diabetes Research

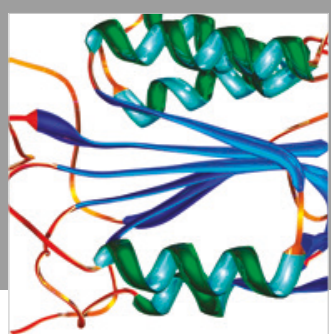

Disease Markers
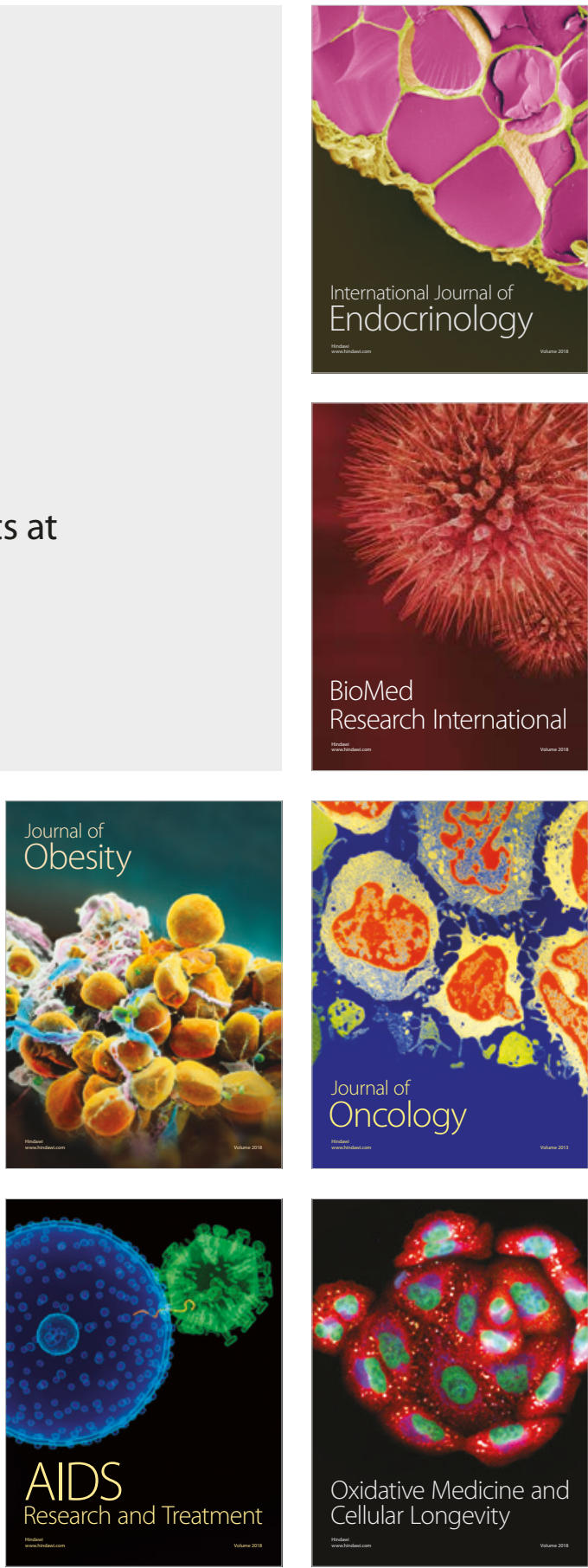